\title{
Bei der Prävention kein Honorar liegen lassen
}

Die Nr. 32880 EBM steht für die Laborpauschale für Urinuntersuchungen im Zusammenhang mit der Erbringung der Gesundheitsuntersuchung unter Nutzung eines Teststreifens. Sie wird als Laborleistung fix mit 50 Cent vergütet. Zusammen mit dem Check-up nach Nr. 01732 ergibt sich so ein extrabudgetäres Honorar von 32,02 Euro. Die Abrechnung ist jedes zweite Jahr möglich - genau wie beim Hautkrebsscreening nach Nr. 01745, das in Kombination weitere 22,33 Euro einbringt.

\section{MMW-KOMMENTAR}

Auch an die Nr. 01740 für die Beratung zur Früherkennung des kolorektalen Karzinoms sollte man denken. Laut Krebsfrüherkennungs-Richtlinien soll der Arzt die Versicherten möglichst frühzeitig nach Vollendung des 50. Lebensjahres einmalig eingehend über das Gesamtprogramm zur Früherkennung des kolorektalen Karzinoms informieren und beraten. Diese erste Beratung ist nicht gesondert abrechnungsfähig, sondern mit den Versicherten- bzw. Grundpauschalen abgegolten. Möglichst bald ab dem Alter von
55 Jahren sollen die Versicherten dann aber eine weitere Beratung erhalten. Dabei geht es um Häufigkeit und Bild der Erkrankung sowie um Ziele, Konzeption, Effektivität (Sensitivität, Spezifität), Wirksamkeit und Nachteile (Belastungen, Risiken) der Früherkennungsuntersuchungen. Auch die Vorgehensweise bei einem positiven Befund wird besprochen. Diese Leistung wird nur einmal im Leben des Patienten mit 10,75 Euro vergütet - extrabudgetär. Im Zeitalter der EDV sollte hier aber wenigstens eine Abdeckung nahe 100\% erreichbar sein.

\section{Separaten Sprechstundenbedarf für Flüchtlinge vorhalten}

_Viele Flüchtlinge mit akuten Beschwerden erhalten einen „grünen“ Abrechnungsschein und werden zu einem niedergelassenen Arzt geschickt. Wer unter das Asylbewerberleistungsgesetz fällt, ist nicht im Rahmen der GKV versichert. Er erhält in den ersten 15 Monaten meist einmal im Quartal einen Behandlungsschein von der zuständigen Behörde, meist dem Sozialamt. Erst dann darf er einen Arzt aufsuchen. Das Gesetz beschränkt in dieser Zeit die Gesundheitsleistungen für Asylbewerber ( $\$ 4$ in Verbindung mit $\$ 2$ Abs. 1 AsylbLG) auf die Behandlung akuter Erkrankungen und von Schmerzzuständen. Aber auch chronische Erkrankungen dürfen grundsätzlich adäquat medizinisch behandelt werden.

Gemäß $\$ 6$ AsylbLG können darüber hinaus sonstige Leistungen gewährt werden, wenn sie zur Sicherung der Gesundheit im Einzelfall unerlässlich sind. Vor einer Überweisung zum Facharzt oder individuellen Therapien ist in der Regel eine erneute Vorsprache bei der Behörde nötig. Gesetzlich gefordert ist dies nicht, man sollte aber klären, ob das Sozialamt die Kosten übernimmt.

\section{MMW-KOMMENTAR}

In diesem Rahmen sind Arznei- und Verbandmittel - auch nicht verschreibungspflichtige Arzneimittel - sowie sonstige zur Genesung, Besserung oder Linderung von Krankheiten oder deren Folgen erforderliche Leistungen (z. B. Heilmittel) zulasten des Sozialamts verordnungsfähig. Eine Zuzahlungspflicht besteht nicht. Die Zuzahlungsbefreiung ergibt sich aus dem Gesetz, sie braucht nicht gesondert auf dem grünen Schein vermerkt zu sein. Zur Absicherung des Asylbewerbers empfiehlt sich aber das Ankreuzen des Feldes "Gebühr frei" auf dem Rezept.

Die Verordnungskosten unterliegen nicht der Wirtschaftlichkeitsprüfung. Impfstoffe müssen auf den Namen des Patienten auf Muster 16 (Kassenrezept) zulasten des Sozialhilfeträ-

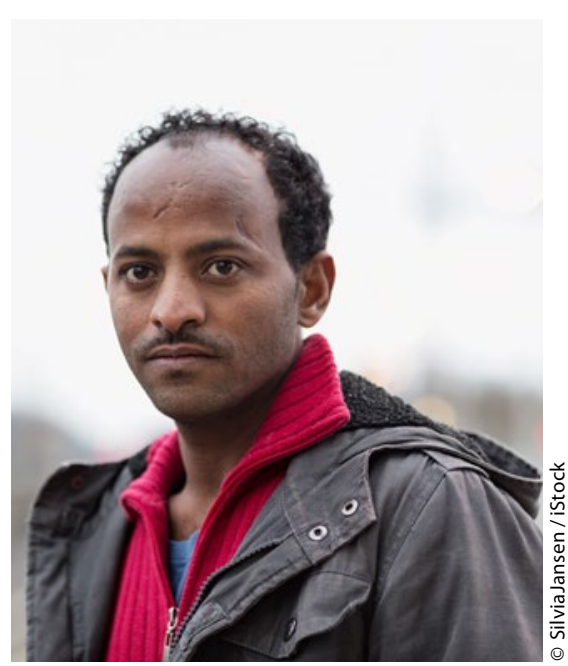

15 Monate lang brauchen Asylbewerber den „grünen“ Schein vom Sozialamt.

gers verordnet werden und dürfen nicht dem Sprechstundenbedarf entnommen werden. Der Sprechstundenbedarf für behandelte Asylbewerber darf ebenfalls nicht dem für die GKV entnommen werden, sondern muss dem Sozialamt in Rechnung gestellt werden. Nach Ablauf der 15 Monate besteht dann allerdings Anspruch auf eine Versorgung auf GKV-Niveau - auch beim Bezugsweg des Sprechstundenbedarfs. 